\title{
Detection of Antibody in Dogs with Blastomycosis Using Blastomyces dermatitidis Yeast Phase Lysate Antigens
}

\author{
Bryn C. Kennell ${ }^{1}$, Gene M. Scalarone1, Lilliana Hoyos-Carvajal2 ${ }^{2}$ Moytri RoyChowdhury ${ }^{1 *}$ \\ ${ }^{1}$ Department of Biological Sciences, Idaho State University, 650 Memorial Dr., Gale Life Sciences Building, Pocatello, ID, USA \\ ${ }^{2}$ Universidad Nacional de Colombia, Sede Medellín, Colombia \\ Email: *roycmoyt@isu.edu
}

How to cite this paper: Kennell, B.C., Scalarone, G.M., Hoyos-Carvajal, L. and RoyChowdhury, M. (2017) Detection of Antibody in Dogs with Blastomycosis Using Blastomyces dermatitidis Yeast Phase Lysate Antigens. Open Journal of Veterinary Medicine, 7, 111-119.

https://doi.org/10.4236/ojvm.2017.79011

Received: July 10, 2017

Accepted: September 27, 2017

Published: September 30, 2017

Copyright $\odot 2017$ by authors and Scientific Research Publishing Inc. This work is licensed under the Creative Commons Attribution International License (CC BY 4.0).

http://creativecommons.org/licenses/by/4.0/

(c) $\underset{\mathrm{EY}}{(7)}$ Open Access

\begin{abstract}
The objective of our study was to compare two $B$. dermatitidis yeast phase lysate antigens [ERC-2, dog Wisconsin; 85, soil Georgia, ATCC 56,920] for detecting antibody in 38 serum specimens [pre-treatment, 30-day, and 60-day post treatment] from dogs with diagnosed blastomycosis. The mean absorbance values obtained with the two antigens $(N=38)$ were ERC- $2=2.359$ and $85=2.189$. The mean absorbance values when the sera were divided into the three treatment groups were as follows pre-treatment: Isolate ERC-2 had an absorbance value of 2.418; Isolate $85 \mathrm{had}$ an absorbance value of 2.688, 30-day post treatment: ERC-2 had an absorbance value of 2.452; 85 had an absorbance value of 2.303 and 60-day post treatment: ERC-2 had an absorbance value of 2.150; 85 had an absorbance value of 2.073 with the mean absorbance values of all treatment groups were ERC-2: 2.229 and 85: 2.141. This study indicates the potential for further evaluations of the two lysate antigens with regard to antibody detection in dog sera with the ERC-2 reagent slightly more reactive than the 85 lysate antigen.
\end{abstract}

\section{Keywords}

Blastomyces Dermatitidis, Yeast Phase Lysate Antigen, Blastomycosis

\section{Introduction}

Blastomycosis is a systemic mycosis that can prove fatal, particularly among the immunocompromised. Blastomycosis is caused by the inhalation of the thermally dimorphic fungal agents Blastomyces dermatitidis. A common reservoir of $B$. dermatitidis is moist soil and decaying matter, especially in en- 
demic areas, like the southeastern and north-central regions of the United States and areas of Canada bordering the states of Wisconsin and Minnesota [1] [2] [3]. Though it has never been directly observed growing in nature, it is thought to grow there as a cottony white mold, similar to the growth seen in artificial culture at $25^{\circ} \mathrm{C}\left(77^{\circ} \mathrm{F}\right)$.

A major virulence factor of $B$. dermatitidis is its ability to convert from a mycelial state at room temperature, to its parasitic state at $37^{\circ} \mathrm{C}$. B. dermatitidis is found in a mycelial state in nature, but once in the lungs, the airborne filaments convert into a broad-based yeast form. The clinical manifestations of blastomycosis range from an asymptomatic infection of the lungs to meningitis. Although the symptoms of blastomycosis are often self-resolving, the primary acute infection may evolve into a chronic form and may even disseminate [4]. These severe forms of blastomycosis can be fatal, especially if treatment is delayed.

The symptoms of blastomycosis mimic various viral and bacterial infections; therefore, the development of more efficient tools would aid in the laboratory diagnosis of blastomycosis. Histological determinations and culturing have been successful in some instances; however, the methods are often time-consuming and expensive [4] [5] [6].

Although there have been promising findings, more research is recommended in order to further test the specificity and sensitivity of various lysate antigens in detecting B. dermatitidis [7] [8] [9] [10]. Blastomycosis is generally readily treatable with systemic antifungal drugs once it is correctly diagnosed; however, delayed diagnosis is very common except in highly endemic areas. The current study investigated the efficacy of two yeast-phase lysate antigens in detecting $B$. dermatitidis antibodies in various dog sera.

\section{Materials and Methods}

\subsection{Lysate Antigens}

Two yeast lysate antigens were prepared from dog (ERC-2, Wisconsin) and soil (85, Georgia) isolates of $B$. dermatitidis. Each of the isolates was prepared by a method similar to one that was previously used for the production of yeast lysate antigen from Histoplasma capsulatum [11] [12] [13] and modified in our laboratory for $B$. dermatitidis lysate antigen production [7].

Mycelial phase cultures were converted to yeast cells by culturing at $37^{\circ} \mathrm{C}$ on BHI agar. Yeast phase lysate reagents were prepared using a method similar to one that was previously described for the production of antigen from Histoplasma capsulatum [7] and modified in our laboratory for B. dermatitidis lysate antigen production [7]. The yeast phase cells were grown for 7 days at $37^{\circ} \mathrm{C}$ in a chemically defined medium in an incubator shaker, harvested by centrifugation $(700 \times$ g; $5 \mathrm{~min}$ ), followed by washing with distilled water, re-suspended in distilled water and then allowed to lyse for 7 days at $37^{\circ} \mathrm{C}$ in water with shaking. The preparations were centrifuged, filter sterilized, and Merthiolate was added $(1: 10,000)$ and the preparations were stored at $4^{\circ} \mathrm{C}$. Protein assays were per- 
formed on the lysates using the BCA Protein Assay Kit (Pierce Chemical Company, Rockford, IL) and dilutions of the antigenic reagents used in the assays were based on protein concentration.

\subsection{Serum Specimens}

Thirty-eight serum specimens from dogs with diagnosed blastomycosis were provided by Dr. A.M. Legendre (University of Tennessee College of Veterinary Medicine, Knoxville, Tennessee).

\subsection{Enzyme Linked Immunosorbent Assay (ELISA)}

The ability of each yeast lysate reagent to detect antibodies in the above serum specimens was determined using the indirect enzyme-linked immunosorbent assay (ELISA) [7] [8] [9] [10]. We followed the methods of M. Shepherd et al. 2014 [14] with only three minor changes. Each lysate antigen was diluted (1000 ng of protein $/ \mathrm{mL}$ ) in a carbonate-bicarbonate coating buffer ( $\mathrm{pH} 9.6)$ and then added to triplicate wells $(100 \mu \mathrm{L})$ of a Greiner 96-well microdilution plate (Phoenix Research Products, Candler NC). The plates were incubated overnight at $4^{\circ} \mathrm{C}$ in a humid chamber and washed three times with phosphate buffered saline containing $0.15 \%$ Tween 20 (PBS-T). The serum specimens (1:5000 dilution; $100 \mu \mathrm{L}$ ) were added to the microdilution plate wells and incubated for $30 \mathrm{~min}$ at $37^{\circ} \mathrm{C}$ in a humid chamber. Following the addition of the goat anti-dog IgG $(\mathrm{H} \&$ L) peroxidase conjugate (Kirkegaard and Perry (KPL), Maryland Gaithersburg, $\mathrm{MD}$ ), the plates were again washed and $100 \mu \mathrm{L}$ of Sure Blue Reserve TMB peroxidase substrate (KPL) was added to each well and incubated for approximately $2 \mathrm{~min}$ at room temperature.

The reaction was stopped by the addition of Stop Solution (KPL) and the absorbance was read at $450 \mathrm{~nm}$ using s Bio-Tek Synergy HT reader.

\section{Results}

ERC-2 lysate antigen exhibited the greatest mean absorbance value (2.359) when the 38 serum specimens from dogs with blastomycosis were assayed using the ELISA as compared to a mean absorbance value of 2.189 with the 85 lysate antigen (Figure 1). In contrast a slightly greater mean absorbance value of 2.688 was obtained with the 85 lysate as compared to a value of 2.418 with the ERC-2 reagent with the pre-treatment sera (Figure 2). The mean absorbance values obtained when the 30-day post-treatment sera were assayed were 2.452 with the ERC-2 antigen and 2.303 with the 85 antigen (Figure 3). The ERC-2 lysate exhibited a similar pattern of reactivity with the 60-day post-treatment serum specimens with a mean absorbance value of 2.150 versus a value of 2.073 with the 85 lysate (Figure 4). A heatmap which was generated using R studio software with GG plot indicated that the amount of antibody detected by the two antigens (ERC-2 and 85) decreased from pretreatment to 60 days post treatment (Figure 5). 


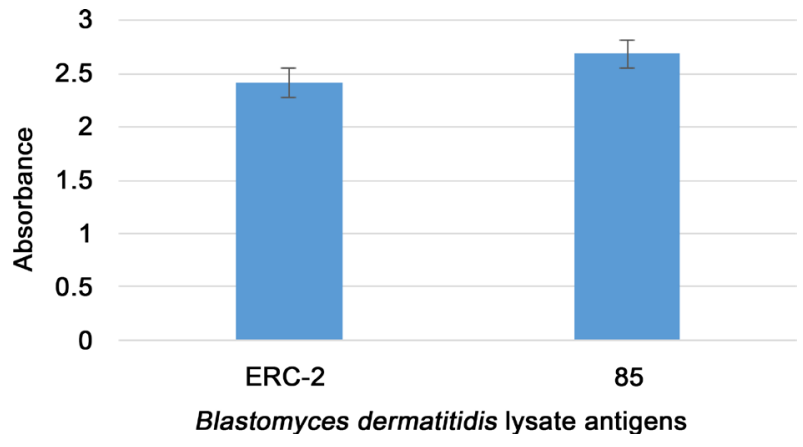

Figure 1. Antibody detection in 38 dog serum specimens with $B$. dermatitidis ERC-2 and 85 lysate antigens.

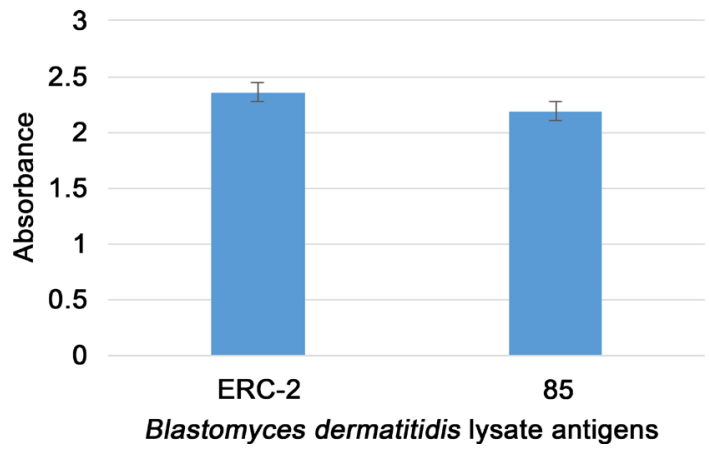

Figure 2. Antibody detection in 17 pre-treatment dog serum specimens with B. dermatitidis ERC-2 and 85 lysate antigens.

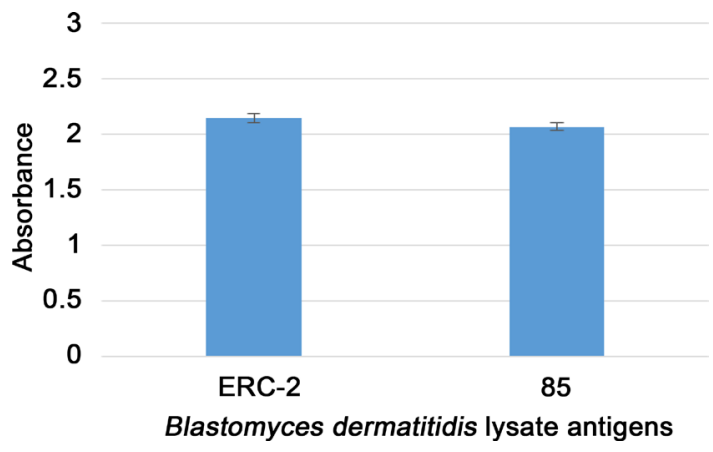

Figure 3. Antibody detection in nine 30 day post-treatment dog serum specimens with $B$. dermatitidis ERC-2 and 85 lysate antigens.

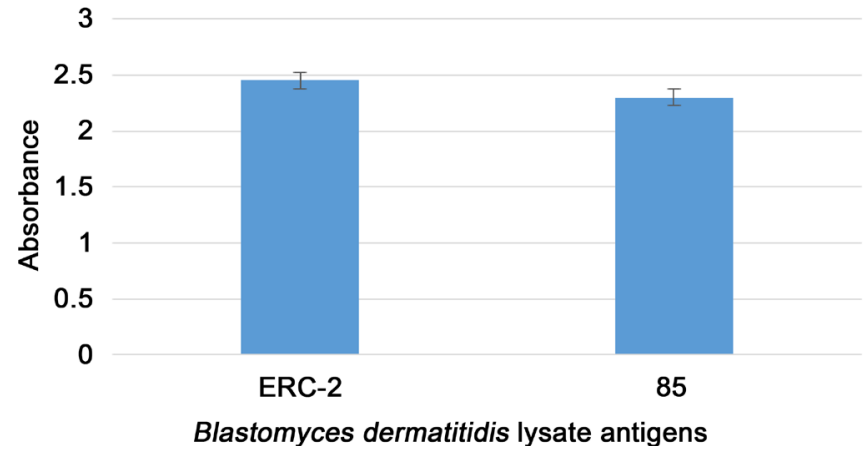

Figure 4. Antibody detection in 1260 day post-treatment dog serum specimens with $B$. dermatitidis ERC-2 and 85 lysate antigens. 


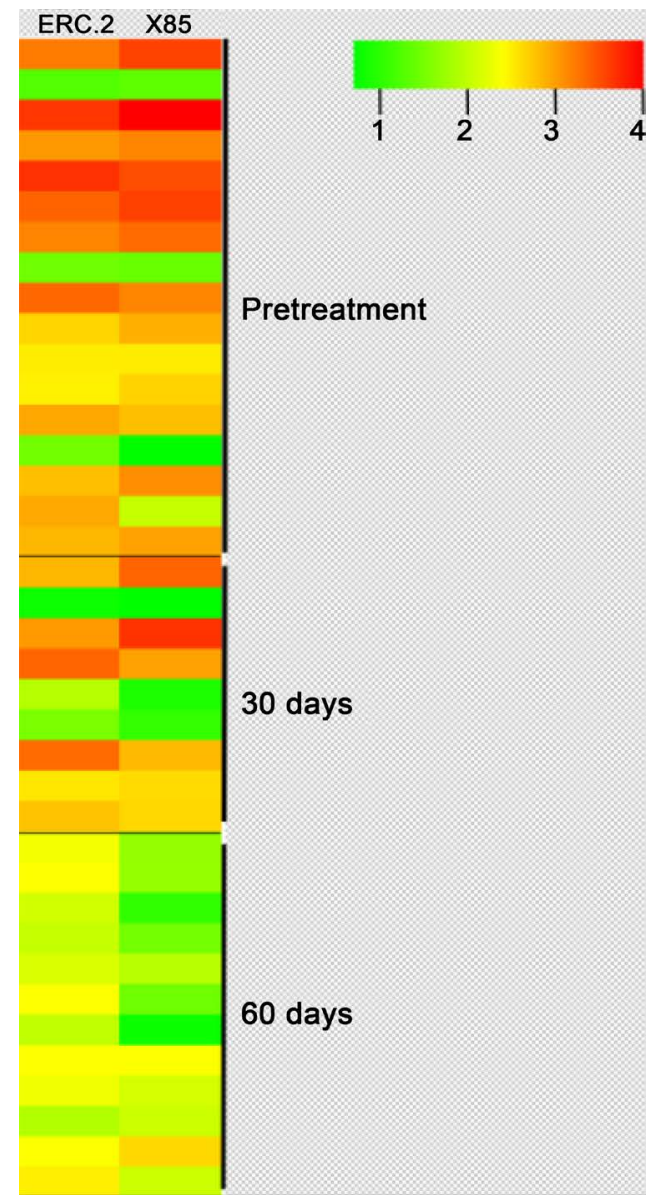

Figure 5. Heatmap showing decrease in the detection of antibody from pretreatment to 60 days post treatment.

\section{Discussion}

Blastomyces dermatitidis is the asexual state of Ajellomyces dermatitidis. A. dermatitidis is the sexual stage of the organism. Production of a sexual spore requires fusion of the nucleus of a positive type with a negative, the so-called heterothallic property; both mating types are equally capable of causing infection [15].

Two serotypes of $B$. dermatitidis have been identified based upon the presence or absence of the A antigen. In a study of 102 isolates predominantly from North America, all of the North American isolates reacted with anti-A antibody, while 11 of 12 isolates from Africa failed to react. Serologic differences in B. dermatitidis isolates from different geographic location in the United States and Africa have been detected using an enzyme-linked immunoassay, indicating that different genotypic groups exist [15].

Phylogenetic analysis of 78 clinical and environmental isolates of $B$. dermatitidis from different geographic regions has revealed two distinct species of the fungus, B. dermatitidis and B. gilchristii. B. gilchristii was isolated from two North American locations known to be hyperendemic for blastomycosis, the Kenora region of Ontario and the Eagle River region of Wisconsin. The authors 
speculated about whether the high rates of infection in these areas might be due to $B$. gilchristii being a more pathogenic species or whether favorable environmental factors allowed emergence of this species. Additional studies are needed using isolates from other sites to establish the prevalence of B. gilchristii [15].

Our lab for several years has been involved in the preparation and evaluation of $B$. dermatitidis yeast phase lysate antigenic preparations from various isolates of the fungus [7] [8] [9] [10]. Our initial research work on the preparation of these novel $B$. dermatitidis lysate antigens for the immunodiagnosis of blastomycosis had a foundation that was based on earlier work that we were involved with on the preparation of lysate antigens for the detection of delayed dermal hypersensitivity or for antibody detection in coccidioidomycosis and histoplasmosis [11] [12] [13]. In an initial study on the development of lysate antigens for the diagnosis of coccidioidomycosis, spherules of Coccidioides immitis were allowed to lyse in distilled water for 40 days to obtain the optimal reactive reagent [13]. In contrast when the same technique was used for the preparation of lysate antigen for the detection of histoplasmosis, it was determined that a one-day period of lysis of Histoplasma capsulatum yeast cells was optimal for the producing a reagent that was useful for skin-testing (delayed dermal hypersensitivity) as well for the detection of antibodies in histoplasmosis using the ELISA [11] [12]. When we applied those parameters to the development of yeast lysate preparations for the diagnosis of blastomycosis, it was determined that a seven-day period of lysis of the yeast cells was optimal for the preparation of a lysate antigen for antibody detection [7].

Most of our immunodiagnostic comparative studies have utilized the indirect ELISA for the detection of antibodies in serum specimens from immunized rabbits or from dogs with diagnosed blastomycosis. The enzyme immunoassay offers a great deal more sensitivity than standard serologic methods that have been used in the past including the immunodiffusion and complement fixation assays [1] [7] [11] [16] [17]. We have performed comparative evaluations of lysates prepared from human, animal and environmental $B$. dermatitidis isolates and encouraging results from those studies have prompted us to perform additional studies with lysates from other isolates of the fungus. The primary objective of this present study was to compare yeast lysate preparations from two isolates of B. dermatitidis (ERC-2, dog, Wisconsin) and 85 (soil, Georgia, ATCC 56,920) for their ability to detect antibodies in 39 serum specimens from dogs with blastomycosis. The data from the study indicated that both $B$. dermatitidis yeast lysate reagents were able to detect antibodies in the serum specimens from dogs with blastomycosis. The optimal degree of reactivity was shown with the ERC-2 antigen prepared from the dog isolate from Wisconsin when compared to the 85 lysate prepared from the soil isolate from Georgia.

The serum specimens were obtained from dogs prior to treatment (17) and at 30-day (9) and 60-day (12) intervals post treatment with itraconazole (Dr. A.M. Legendre). The ELISA results indicated that with the ERC-2 antigen the mean 
absorbance values were similar with the pre-treatment and 30-day sera (2.418, 2.452 ), but the mean absorbance value with the 60-day specimens was 2.150 . Antibody detection with the 85 lysate antigen showed a decrease in reactivity from the pre-treatment to the 30-day and 60-day post treatment specimens with mean absorbance values of 2.688, 2.303 and 2.073 respectively. These antibody detection values are what one would assume following treatment. This study indicates the potential of both of these $B$. dermatitidis antigenic reagents for antibody detection in the ELISA and additional comparative studies are warranted to assess the value of such preparations for the immunodiagnosis of blastomycosis in animals and humans.

Conversion of $B$. dermatitidis to the yeast form induces the expression of an essential virulence factor, BAD-1 (formerly WI-1), a $120 \mathrm{kd}$ glycoprotein adhesion and immune modulator of $B$. dermatitidis. The major acquired host defense against $B$. dermatitidis is cellular immunity, which is mediated by antigen-specific $\mathrm{T}$ lymphocytes and lymphokine-activated macrophages. It is to be noted here that our knowledge of the epidemiology of blastomycosis remains incomplete because of the lack of well-characterized antigens for skin testing or for seroepidemiologic studies. Current information on the epidemiology of blastomycosis is based upon clinical reports of sporadic cases in humans and dogs and the study of point source outbreaks of disease [15].

\section{Conclusion}

Our study, which compared 2 lysate antigens with regard to antibody detection in sera from dogs with blastomycosis, indicated that the optimal $B$. dermatitidis antigenic preparation was the lysate prepared from the dog isolate with the ERC-2 antigen when compared to 85 antigen. We also observed that the amount of antibody detected by the two antigens (ERC-2 and 85) decreased from pretreatment to 60 days post treatment.

\section{Funding}

Publication costs for the manuscript was provided by Universidad Nacional de Colombia-Sede Medellín, Colombia; The State Board of Education/Idaho State University STEM program [grant number AHRC38] funded the hourly wages for the student.

\section{Acknowledgements}

This research was supported through the generous contributions through bench work research from several students in the Department of Biological Sciences at Idaho State University, Pocatello, Idaho, USA.

\section{Conflict of Interest}

The authors declare that there is no conflict of interest regarding the publication of this paper. 


\section{References}

[1] DiSalvo, A.F. (1998) "Blastomycosis", In: Collier, L., Ed., Topley and Wilson's Microbiology and Microbial Infections, $9^{\text {th }}$ Edition, Arnold Publishers, London, UK, 337-355.

[2] Pfaller, M.A. and Diekema, D.J. (2010) Epidemiology of Invasive Mycoses in North America. Critical Reviews in Microbiology, 36, 1-53.

https://doi.org/10.3109/10408410903241444

[3] Benedict, K., Roy, M., Chiller, T. and Davis, J.P. (2012) Epidemiologic and Ecologic Features of Blastomycosis: A Review. Current Fungal Infection Reports, 6, 327-335. https://doi.org/10.1007/s12281-012-0110-1

[4] Cutler, J.E., Deepe, G.S. Jr and Klein, B.S. (2007) Advances in Combating Fungal Diseases: Vaccines on the Threshold. Nature Review of Microbiology, 5, 13-18, https://doi.org/10.1038/nrmicro1537

[5] Saccente, M. and Woods, G.L. (2010) Clinical and Laboratory Update on Blastomycosis. Clinical Microbiology Reviews, 23, 367-381. https://doi.org/10.1128/CMR.00056-09

[6] Bradsher, R.W., Chapman, S.W. and Pappas, P.G. (2003) Blastomycosis. Infectious Disease Clinics of North America, 17, 21-40. https://doi.org/10.1016/S0891-5520(02)00038-7

[7] Johnson, S.M. and Scalarone, G.M. (1989) Preparation and ELISA Evaluation of Blastomyces dermatitidis Yeast Phase Lysate Antigens. Diagnostic Microbiology and Infectious Diseases, 11, 81-86.

[8] Braun, J., Counsell, K., Davis, C., Twamley, S. and Scalarone, G.M. (2014) Antigen Detection in Urine Specimens from Dogs with Blastomycosis: Comparison of Rabbit Antibodies Prepared from a Human and Dog Isolate of Blastomyces dermatitidis. US Open Infectious Diseases \& Immunity Journal, 1, 1-6.

[9] Poole, J.B., Cox, R.T., Cobb, E.R., Boyd, A.R. and Scalarone, G.M. (2014) Comparison of Antibody Detection with Lysate Antigens Prepared from Human and Dog Isolates of Blastomyces dermatitidis. US Open Infectious Diseases \& Immunity Journal, 1, 1-5.

[10] Rayner, C., Blackwell, T., Hine, T., Court, J. and Scalarone, G.M. (2014) A Comparative Study using 28 Blastomyces dermatitidis Yeast Lysate Antigens for Antibody Detection in Serum Specimens from Immunized Rabbits and Dogs with Blastomycosis. US Open Infectious Diseases \& Immunity Journal, 1, 1-8.

[11] Levine, H.B., Scalarone, G.M., Campbell, G.D., Graybill, R.C. and Chaparas, S.D. (1979) Histoplasmin-CYL, A Yeast Phase Reagent in Skin Test Studies in Humans. American Review of Respiratory Diseases, 119, 629-636.

[12] Scalarone, G.M., Levine, H.B. and Chaparas, S.D. (1978) Delayed Hypersensitivity Responses of Experimental Animals to Histoplasmin from the Yeast and Mycelial Phases of Histoplasma capsulatum. Infection and Immunity, 21, 705-713.

[13] Levine, H.B., Scalarone, G.M. and Chaparas, S.D. (1977) Preparation of Fungal Antigens and Vaccines: Studies on Coccidioides immitis and Histoplasma capsulatum. Contributions to Microbiology and Immunology, 3, 106-125.

[14] Shepherd, M., Lutes, M. and Scalarone, G.M. (2014) Blastomyces dermatitidis Antiabody Responses in Serial Serum Specimens from Dogs with Blastomycosis: Comparison of Different Yeast Lysate Antigens. Journal of Biosciences and Medicines, 2, 67-73.

[15] Bradsher, Jr, R.W. Mycology, Pathogenesis, and Epidemiology of Blastomycosis. 
http://cursoenarm.net/UPTODATE/contents/mobipreview.htm?23/6/23662

[16] McKinnell, J.A. and Pappas, P.G. (2009) Blastomycosis: New Insights into Diagnosis, Prevention, and Treatment. Clinical Chest Medicine, 30, 227-239. https://doi.org/10.1016/j.ccm.2009.02.003

[17] Vyas, K.S., Bariola, J.R. and Bradsher, R.W. (2008) Advances in the Serodiagnosis of Blastomycosis. Current Fungal Infection Reports, 2, 227-231.

https://doi.org/10.1007/s12281-008-0033-Z

Submit or recommend next manuscript to SCIRP and we will provide best service for you:

Accepting pre-submission inquiries through Email, Facebook, LinkedIn, Twitter, etc. A wide selection of journals (inclusive of 9 subjects, more than 200 journals)

Providing 24-hour high-quality service

User-friendly online submission system

Fair and swift peer-review system

Efficient typesetting and proofreading procedure

Display of the result of downloads and visits, as well as the number of cited articles Maximum dissemination of your research work

Submit your manuscript at: http://papersubmission.scirp.org/

Or contact ojvm@scirp.org 